\title{
Search for Promptly Produced Heavy Quarkonium States in Hadronic Z Decays
}

\author{
DELPHI Collaboration
}

\begin{abstract}
A search has been made for direct production of heavy quarkonium states in more than 3 million hadronic $Z^{0}$ decays in the 1991-1994 DELPHI data. Prompt $J / \psi, \psi(2 S)$ and $\Upsilon$ candidates have been searched for through their leptonic decay modes using criteria based on the kinematics and decay vertex positions. New upper limits are set at the $90 \%$ confidence level for $\operatorname{Br}\left(Z^{0} \rightarrow(Q \bar{Q}) X\right) / \operatorname{Br}\left(Z^{0} \rightarrow\right.$ hadrons $)$ for various strong production mechanisms of $J / \psi$ and $\Upsilon$; these range down to $0.9 \times 10^{-4}$. The limits are set in the presence of a small excess $(\sim 1 \%$ statistical probability of a background fluctuation) in the sum of candidates from prompt $J / \psi, \psi(2 S), \Upsilon(1 S), \Upsilon(2 S)$ and $\Upsilon(3 S)$ relative to the estimated background.
\end{abstract}


P.Abreu ${ }^{21}$, W.Adam ${ }^{50}$, T.Adye ${ }^{37}$, E.Agasi ${ }^{31}$, I.Ajinenko ${ }^{42}$, R.Aleksan ${ }^{39}$, G.D.Alekseev ${ }^{16}$, P.P.Allport ${ }^{22}$, S.Almehed $^{24}$, S.J.Alvsvaag ${ }^{4}$, U.Amaldi ${ }^{9}, \quad$ S.Amato $^{47}$, A.Andreazza ${ }^{28}$, M.L.Andrieux ${ }^{14}$, P.Antilogus ${ }^{9}$, W-D.Apel ${ }^{17}$, Y.Arnoud ${ }^{39}$, B.Asman ${ }^{44}$, J-E.Augustin ${ }^{19}$, A.Augustinus ${ }^{31}$, P.Baillon ${ }^{9}$, P.Bambade ${ }^{19}$, F.Barao $^{21}$, R.Barate $^{14}$, G.Barbiellini ${ }^{46}$, D.Y.Bardin ${ }^{16}$, G.J.Barker ${ }^{35}$, A.Baroncelli ${ }^{40}$, O.Barring ${ }^{24}$, J.A.Barrio ${ }^{26}$, W.Bartl ${ }^{50}$, M.J.Bates ${ }^{37}$, M.Battaglia ${ }^{15}$, M.Baubillier ${ }^{23}$, J.Baudot ${ }^{39}$, K-H.Becks ${ }^{52}$, M.Begalli ${ }^{6}$, P.Beilliere ${ }^{8}$, Yu.Belokopytov ${ }^{9,53}$, K.Belous ${ }^{42}$, A.C.Benvenuti ${ }^{5}$, M.Berggren ${ }^{47}$, D.Bertrand ${ }^{2}$, F.Bianchi ${ }^{45}$, M.Bigi $^{45}$, M.S.Bilenky ${ }^{16}$, P.Billoir ${ }^{23}$, D.Bloch ${ }^{10}$, M.Blume ${ }^{52}$, S.Blyth ${ }^{35}$, V.Bocci ${ }^{38}$, T.Bolognese ${ }^{39}$, M.Bonesini ${ }^{28}$, W.Bonivento ${ }^{28}$, P.S.L.Booth ${ }^{22}$, G.Borisov ${ }^{42}$, C.Bosio ${ }^{40}$, S.Bosworth ${ }^{35}$, O.Botner ${ }^{48}$, B.Bouquet $^{19}$, C.Bourdarios ${ }^{9}$, T.J.V.Bowcock ${ }^{22}$, M.Bozzo ${ }^{13}$, P.Branchini ${ }^{40}$, K.D.Brand ${ }^{36}$, T.Brenke ${ }^{52}$, R.A.Brenner ${ }^{15}$, C.Bricman $^{2}$, L.Brillault ${ }^{23}$, R.C.A.Brown ${ }^{9}$, P.Bruckman ${ }^{18}$, J-M.Brunet ${ }^{8}$, L.Bugge ${ }^{33}$, T.Buran ${ }^{33}$, T.Burgsmueller ${ }^{52}$, P.Buschmann ${ }^{52}$, A.Buys ${ }^{9}$, M.Caccia $^{28}$, M.Calvi ${ }^{28}$, A.J.Camacho Rozas ${ }^{41}$, T.Camporesi $^{9}$, V.Canale ${ }^{38}$, M.Canepa ${ }^{13}$, K.Cankocak ${ }^{44}$, F.Cao $^{2}, \quad$ F.Carena ${ }^{9}$, P.Carrilho ${ }^{47}$, L.Carroll ${ }^{22}$, C.Caso ${ }^{13}$, M.V.Castillo Gimenez ${ }^{49}$, A.Cattai ${ }^{9}$, F.R.Cavallo ${ }^{5}$, L.Cerrito ${ }^{38}$, V.Chabaud ${ }^{9}$, Ph.Charpentier ${ }^{9}$, L.Chaussard $^{25}$, J.Chauveau $^{23}$, P.Checchia ${ }^{36}$, G.A.Chelkov ${ }^{16}$, R.Chierici ${ }^{45}$, P.Chliapnikov ${ }^{42}$, P.Chochula ${ }^{7}$, V.Chorowicz ${ }^{9}$, V.Cindro ${ }^{43}$, P.Collins ${ }^{9}$, J.L.Contreras ${ }^{19}$, R.Contri ${ }^{13}$, E.Cortina ${ }^{49}$, G.Cosme ${ }^{19}$, F.Cossutti ${ }^{46}$, H.B.Crawley ${ }^{1}$, D.Crennell $^{37}$, G.Crosetti ${ }^{13}$, J.Cuevas Maestro ${ }^{34}$, S.Czellar ${ }^{15}$, E.Dahl-Jensen ${ }^{29}$, J.Dahm ${ }^{52}$, B.Dalmagne ${ }^{19}$, M.Dam $^{29}$, G.Damgaard ${ }^{29}$, P.D.Dauncey ${ }^{37}$, M.Davenport ${ }^{9}$, W.Da Silva ${ }^{23}$, C.Defoix ${ }^{8}$, A.Deghorain ${ }^{2}$, G.Della Ricca ${ }^{46}$, P.Delpierre ${ }^{27}$, N.Demaria ${ }^{35}$, A.De Angelis ${ }^{9}$, H.De Boeck ${ }^{2}$, W.De Boer ${ }^{17}$, S.De Brabandere ${ }^{2}$, C.De Clercq $^{2}$, C.De La Vaissiere ${ }^{23}$, B.De Lotto ${ }^{46}$, A.De Min ${ }^{36}$, L.De Paula ${ }^{47}$, C.De Saint-Jean ${ }^{39}$, H.Dijkstra ${ }^{9}$, L.Di Ciaccio ${ }^{38}$, F.Djama ${ }^{10}$, J.Dolbeau ${ }^{8}$, M.Donszelmann ${ }^{9}$, K.Doroba ${ }^{51}$, M.Dracos ${ }^{10}$, J.Drees ${ }^{52}$, K.-A.Drees ${ }^{52}$, M.Dris $^{32}$, Y.Dufour ${ }^{9}$, F.Dupont ${ }^{14}$, D.Edsall ${ }^{1}$, R.Ehret ${ }^{17}$, G.Eigen ${ }^{4}$, T.Ekelof ${ }^{48}$, G.Ekspong ${ }^{44}$, M.Elsing ${ }^{52}$, J-P.Engel $^{10}$, N.Ershaidat ${ }^{23}$, B.Erzen ${ }^{43}$, M.Espirito Santo ${ }^{21}$, E.Falk ${ }^{24}$, D.Fassouliotis ${ }^{32}$, M.Feindt ${ }^{9}$, A.Ferrer ${ }^{49}$, T.A.Filippas ${ }^{32}$, A.Firestone ${ }^{1}$, P.-A.Fischer ${ }^{10}$, H.Foeth ${ }^{9}$, E.Fokitis ${ }^{32}$, F.Fontanelli ${ }^{13}$, F.Formenti $^{9}$, B.Franek ${ }^{37}$, P.Frenkiel $^{8}$, D.C.Fries ${ }^{17}$, A.G.Frodesen ${ }^{4}$, R.Fruhwirth ${ }^{50}$, F.Fulda-Quenzer ${ }^{19}$, J.Fuster ${ }^{49}$, A.Galloni ${ }^{22}$, D.Gamba ${ }^{45}$, M.Gandelman $^{6}$, C.Garcia $^{49}$, J.Garcia ${ }^{41}$, C.Gaspar $^{9}$, U.Gasparini $^{36}$, Ph.Gavillet $^{9}$, E.N.Gazis ${ }^{32}$, D.Gele ${ }^{10}$, J-P.Gerber ${ }^{10}$, M.Gibbs ${ }^{22}$, R.Gokieli ${ }^{51}$, B.Golob ${ }^{43}$, G.Gopal ${ }^{37}$, L.Gorn ${ }^{1}$, M.Gorski ${ }^{51}$, Yu.Gouz ${ }^{45,42}$, V.Gracco $^{13}$, E.Graziani ${ }^{40}$, G.Grosdidier ${ }^{19}$, K.Grzelak ${ }^{51}$, S.Gumenyuk ${ }^{28,53}$, P.Gunnarsson ${ }^{44}$, M.Gunther ${ }^{48}$,

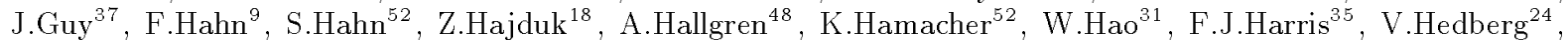
R.Henriques $^{21}$, J.J.Hernandez ${ }^{49}$, P.Herquet ${ }^{2}$, H.Herr ${ }^{9}$, T.L.Hessing ${ }^{9}$, E.Higon ${ }^{49}$, H.J.Hilke ${ }^{9}$, T.S.Hill ${ }^{1}$, S-O.Holmgren ${ }^{44}$, P.J.Holt ${ }^{35}$, D.Holthuizen ${ }^{31}$, S.Hoorelbeke ${ }^{2}$, M.Houlden ${ }^{22}$, J.Hrubec ${ }^{50}$, K.Huet ${ }^{2}$, K.Hultqvist ${ }^{44}$, J.N.Jackson ${ }^{22}$, R.Jacobsson ${ }^{44}$, P.Jalocha ${ }^{18}$, R.Janik ${ }^{7}$, G.Jarlskog ${ }^{24}$, P.Jarry ${ }^{39}$, B.Jean-Marie ${ }^{19}$, E.K.Johansson ${ }^{44}$, L.Jonsson ${ }^{24}$, P.Jonsson ${ }^{24}$, C.Joram ${ }^{9}$, P.Juillot ${ }^{10}$, M.Kaiser ${ }^{17}$, F.Kapusta ${ }^{23}$, K.Karafasoulis ${ }^{11}$, M.Karlsson ${ }^{44}$, E.Karvelas ${ }^{11}$, S.Katsanevas ${ }^{3}$, E.C.Katsoufis ${ }^{32}$, R.Keranen ${ }^{44}$, B.A.Khomenko ${ }^{16}$, N.N.Khovanski ${ }^{16}{ }$, B.King ${ }^{22}$, N.J.Kjaer ${ }^{9}$, H.Klein ${ }^{9}$, A.Klovning ${ }^{4}$, P.Kluit ${ }^{31}$, B.Koene ${ }^{31}$, P.Kokkinias ${ }^{11}$, M.Koratzinos ${ }^{9}$, V.Kostioukhine $^{42}$, C.Kourkoumelis ${ }^{3}$, O.Kouznetsov ${ }^{13}$, P.-H.Kramer ${ }^{52}$, M.Krammer ${ }^{50}$, C.Kreuter $^{17}$, J.Krolikowski $^{51}$, I.Kronkvist ${ }^{24}$, Z.Krumstein ${ }^{16}$, W.Krupinski ${ }^{18}$, P.Kubinec ${ }^{7}$, W.Kucewicz ${ }^{18}$, K.Kurvinen ${ }^{15}$, C.Lacasta $^{49}$, I.Laktineh ${ }^{25}$, S.Lamblot ${ }^{23}$, J.W.Lamsa ${ }^{1}$, L.Lanceri ${ }^{46}$, D.W.Lane ${ }^{1}$, P.Langefeld ${ }^{52}$, V.Lapin $^{42}{ }^{4}$, I.Last $^{22}$, J-P.Laugier ${ }^{39}$, R.Lauhakangas ${ }^{15}$, G.Leder ${ }^{50}$, F.Ledroit ${ }^{14}$, V.Lefebure ${ }^{2}$, C.K.Legan ${ }^{1}$, R.Leitner ${ }^{30}$, Y.Lemoigne $^{39}$, J.Lemonne ${ }^{2}$, G.Lenzen ${ }^{52}$, V.Lepeltier ${ }^{19}$, T.Lesiak ${ }^{36}$, D.Liko ${ }^{50}$, R.Lindner ${ }^{52}$, A.Lipniacka ${ }^{36}$, I.Lippi $^{36}$, B.Loerstad ${ }^{24}$, M.Lokajicek ${ }^{12}$, J.G.Loken ${ }^{35}$, J.M.Lopez ${ }^{41}$, A.Lopez-Fernandez ${ }^{9}$, M.A.Lopez Aguera ${ }^{41}$, D.Loukas ${ }^{11}$, P.Lutz ${ }^{39}$, L.Lyons ${ }^{35}$, J.MacNaughton ${ }^{50}$, G.Maehlum ${ }^{17}$, A.Maio ${ }^{21}$, V.Malychev ${ }^{16}$, C.Maocun ${ }^{2}$, J.Marco $^{41}$, B.Marechal ${ }^{47}$, M.Margoni ${ }^{36}$, J-C.Marin ${ }^{9}$, C.Mariotti ${ }^{40}$, A.Markou ${ }^{11}$, T.Maron ${ }^{52}$, C.Martinez-Rivero $^{41}$, F.Martinez-Vidal ${ }^{49}$, S.Marti i Garcia ${ }^{49}$, F.Matorras ${ }^{41}$, C.Matteuzzi ${ }^{28}$, G.Matthiae ${ }^{38}$, M.Mazzucato $^{36}$, M.Mc Cubbin ${ }^{9}$, R.Mc Kay ${ }^{1}$, R.Mc Nulty ${ }^{22}$, J.Medbo ${ }^{48}$, C.Meroni ${ }^{28}$, S.Meyer ${ }^{17}$, W.T.Meyer ${ }^{1}$, A.Miagkov $^{42}$, M.Michelotto ${ }^{36}$, E.Migliore ${ }^{45}$, L.Mirabito ${ }^{25}$, W.A.Mitaroff ${ }^{50}$, U.Mjoernmark ${ }^{24}$, T.Moa ${ }^{44}$, R.Moeller $^{29}$, K.Moenig 9 , M.R.Monge ${ }^{13}$, P.Morettini ${ }^{13}$, H.Mueller ${ }^{17}$, L.M.Mundim ${ }^{6}$, W.J.Murray ${ }^{37}$, B.Muryn $^{18}$, G.Myatt $^{35}$, F.Naraghi ${ }^{14}$, F.L.Navarria ${ }^{5}$, S.Navas ${ }^{49}$, P.Negri ${ }^{28}$, S.Nemecek ${ }^{12}$, W.Neumann ${ }^{52}$, N.Neumeister ${ }^{50}$, R.Nicolaidou ${ }^{3}$, B.S.Nielsen ${ }^{29}$, M.Nieuwenhuizen ${ }^{31}$, V.Nikolaenko ${ }^{10}$, P.Niss ${ }^{44}$, A.Nomerotski ${ }^{36}$, A.Normand ${ }^{35}$, W.Oberschulte-Beckmann ${ }^{17}$, V.Obraztsov ${ }^{42}$, A.G.Olshevski ${ }^{16}$, A.Onofre ${ }^{21}$, R.Orava ${ }^{15}$, K.Osterberg ${ }^{15}$, A.Ouraou $^{39}$, P.Paganini ${ }^{19}$, M.Paganoni ${ }^{9}$, P.Pages ${ }^{10}$, H.Palka ${ }^{18}$, Th.D.Papadopoulou ${ }^{32}$, K.Papageorgiou ${ }^{11}$, L.Pape $^{9}$, C.Parkes ${ }^{35}$, F.Parodi ${ }^{13}$, A.Passeri ${ }^{40}$, M.Pegoraro ${ }^{36}$, L.Peralta ${ }^{21}$, H.Pernegger ${ }^{50}$, M.Pernicka ${ }^{50}$, A.Perrotta $^{5}$, C.Petridou ${ }^{46}$, A.Petrolini ${ }^{13}$, M.Petrovyck ${ }^{28,53}$, H.T.Phillips ${ }^{37}$, G.Piana ${ }^{13}$, F.Pierre ${ }^{39}$, M.Pimenta ${ }^{21}$, M.Pindo $^{28}$, S.Plaszczynski ${ }^{19}$, O.Podobrin ${ }^{17}$, M.E.Pol ${ }^{6}$, G.Polok ${ }^{18}$, P.Poropat ${ }^{46}$, V.Pozdniakov ${ }^{16}$, M.Prest $^{46}$, P.Privitera $^{38}$, N.Pukhaeva ${ }^{16}$, D.Radojicic ${ }^{35}$, S.Ragazzi $^{28}$, H.Rahmani ${ }^{32}$, J.Rames ${ }^{12}$, P.N.Ratoff ${ }^{20}$, A.L.Read ${ }^{33}$, M.Reale ${ }^{52}$, P.Rebecchi ${ }^{19}$, N.G.Redaelli ${ }^{28}$, M.Regler ${ }^{50}$, D.Reid ${ }^{9}$, P.B.Renton ${ }^{35}$, L.K.Resvanis ${ }^{3}$, F.Richard ${ }^{19}$, J.Richardson ${ }^{22}$, J.Ridky ${ }^{12}$, G.Rinaudo ${ }^{45}$, I.Ripp ${ }^{39}$, A.Romero ${ }^{45}$, I.Roncagliolo ${ }^{13}$, P.Ronchese ${ }^{36}$, L.Roos $^{14}$, E.I.Rosenberg ${ }^{1}$, E.Rosso ${ }^{9}$, P.Roudeau ${ }^{19}$, T.Rovelli ${ }^{5}$, W.Ruckstuhl ${ }^{31}$, V.Ruhlmann-Kleider ${ }^{39}$, A.Ruiz ${ }^{41}$, H.Saarikko ${ }^{15}$, Y.Sacquin ${ }^{39}$, A.Sadovsky ${ }^{16}$, G.Sajot ${ }^{14}$, J.Salt ${ }^{49}$, J.Sanchez ${ }^{26}$, M.Sannino ${ }^{13}$, M.Schimmelpfennig ${ }^{17}$, H.Schneider ${ }^{17}$, U.Schwickerath ${ }^{17}$, M.A.E.Schyns ${ }^{52}$, G.Sciolla ${ }^{45}$, F.Scuri ${ }^{46}$, P.Seager ${ }^{20}$, Y.Sedykh ${ }^{16}$, A.M.Segar ${ }^{35}$,

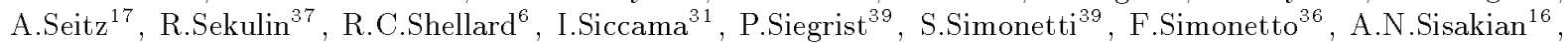
B.Sitar ${ }^{7}$, T.B.Skaali ${ }^{33}$, G.Smadja ${ }^{25}$, N.Smirnov ${ }^{42}$, O.Smirnova ${ }^{16}$, G.R.Smith ${ }^{37}$, R.Sosnowski ${ }^{51}$, D.Souza-Santos ${ }^{6}$, T.Spassov ${ }^{21}$, E.Spiriti ${ }^{40}$, P.Sponholz ${ }^{52}$, S.Squarcia ${ }^{13}$, C.Stanescu ${ }^{40}$, S.Stapnes ${ }^{33}$, I.Stavitski ${ }^{36}$, F.Stichelbaut $^{9}$, A.Stocchi ${ }^{19}$, J.Strauss ${ }^{50}$, R.Strub ${ }^{10}$, B.Stugu ${ }^{4}$, M.Szczekowski ${ }^{51}$, M.Szeptycka ${ }^{51}$, T.Tabarelli ${ }^{28}{ }^{\text {, }}$ 
J.P.Tavernet $^{23}$, O.Tchikilev ${ }^{42}$, A.Tilquin ${ }^{27}$, J.Timmermans ${ }^{31}$, L.G.Tkatchev ${ }^{16}$, T.Todorov ${ }^{10}$, D.Z.Toet ${ }^{31}$, A.Tomaradze $^{2}$, B.Tome ${ }^{21}$, L.Tortora ${ }^{40}$, G.Transtromer ${ }^{24}$, D.Treille ${ }^{9}$, W.Trischuk ${ }^{9}$, G.Tristram ${ }^{8}$, A.Trombini ${ }^{19}{ }^{9}$, C.Troncon $^{28}$, A.Tsirou ${ }^{9}$, M-L.Turluer ${ }^{39}$, I.A.Tyapkin ${ }^{16}$, M.Tyndel ${ }^{37}$, S.Tzamarias ${ }^{22}$, B.Ueberschaer ${ }^{52}$, O.Ullaland ${ }^{9}$, V.Uvarov ${ }^{42}$, G.Valenti ${ }^{5}$, E.Vallazza ${ }^{9}$, C.Vander Velde ${ }^{2}$, G.W.Van Apeldoorn ${ }^{31}$, P.Van Dam ${ }^{31}$, W.K.Van Doninck ${ }^{2}$, J.Van Eldik ${ }^{31}$, N.Vassilopoulos ${ }^{35}$, G.Vegni ${ }^{28}$, L.Ventura ${ }^{36}$, W.Venus ${ }^{37}$, F.Verbeure ${ }^{2}$, M.Verlato $^{36}$, L.S.Vertogradov ${ }^{16}$, D.Vilanova ${ }^{39}$, P.Vincent ${ }^{25}$, L.Vitale ${ }^{46}$, E.Vlasov ${ }^{42}$, A.S.Vodopyanov $^{16}$, V.Vrba $^{12}$, H.Wahlen ${ }^{52}$, C.Walck ${ }^{44}$, M.Weierstall ${ }^{52}$, P.Weilhammer ${ }^{9}$, C.Weiser ${ }^{17}$, A.M.Wetherell ${ }^{9}$, D.Wicke ${ }^{52}$, J.H.Wickens ${ }^{2}$, M.Wielers ${ }^{17}$, G.R.Wilkinson ${ }^{35}$, W.S.C.Williams ${ }^{35}$, M.Winter ${ }^{10}$, M.Witek $^{18}$, K.Woschnagg $^{48}$, K.Yip ${ }^{35}$, O.Yushchenko ${ }^{42}$, F.Zach ${ }^{25}$, C.Zacharatou ${ }^{24}$, A.Zaitsev ${ }^{42}$, A.Zalewska ${ }^{18}$, P.Zalewski ${ }^{51}$, D.Zavrtanik ${ }^{43}$, E.Zevgolatakos ${ }^{11}$, N.I.Zimin ${ }^{16}$, M.Zito ${ }^{39}$, D.Zontar ${ }^{43}$, R.Zuberi ${ }^{35}$, G.C.Zucchelli ${ }^{44}$, G.Zumerle ${ }^{36}$

\footnotetext{
${ }^{1}$ Ames Laboratory and Department of Physics, Iowa State University, Ames IA 50011, USA

${ }^{2}$ Physics Department, Univ. Instelling Antwerpen, Universiteitsplein 1, B-2610 Wilrijk, Belgium and IIHE, ULB-VUB, Pleinlaan 2, B-1050 Brussels, Belgium

and Faculté des Sciences, Univ. de l'Etat Mons, Av. Maistriau 19, B-7000 Mons, Belgium

${ }^{3}$ Physics Laboratory, University of Athens, Solonos Str. 104, GR-10680 Athens, Greece

${ }^{4}$ Department of Physics, University of Bergen, Allégaten 55, N-5007 Bergen, Norway

${ }^{5}$ Dipartimento di Fisica, Università di Bologna and INFN, Via Irnerio 46, I-40126 Bologna, Italy

${ }^{6}$ Centro Brasileiro de Pesquisas Físicas, rua Xavier Sigaud 150, RJ-22290 Rio de Janeiro, Brazil and Depto. de Física, Pont. Univ. Católica, C.P. 38071 RJ-22453 Rio de Janeiro, Brazil and Inst. de Física, Univ. Estadual do Rio de Janeiro, rua São Francisco Xavier 524, Rio de Janeiro, Brazil ${ }^{7}$ Comenius University, Faculty of Mathematics and Physics, Mlynska Dolina, SK-84215 Bratislava, Slovakia

${ }^{8}$ Collège de France, Lab. de Physique Corpusculaire, IN2P3-CNRS, F-75231 Paris Cedex 05, France

${ }^{9} \mathrm{CERN}, \mathrm{CH}-1211$ Geneva 23, Switzerland

${ }^{10}$ Centre de Recherche Nucléaire, IN2P3 - CNRS/ULP - BP20, F-67037 Strasbourg Cedex, France

${ }^{11}$ Institute of Nuclear Physics, N.C.S.R. Demokritos, P.O. Box 60228, GR-15310 Athens, Greece

${ }^{12}$ FZU, Inst. of Physics of the C.A.S. High Energy Physics Division, Na Slovance 2, 180 40, Praha 8, Czech Republic

${ }^{13}$ Dipartimento di Fisica, Università di Genova and INFN, Via Dodecaneso 33, I-16146 Genova, Italy

${ }^{14}$ Institut des Sciences Nucléaires, IN2P3-CNRS, Université de Grenoble 1, F-38026 Grenoble Cedex, France

${ }^{15}$ Research Institute for High Energy Physics, SEFT, P.O. Box 9, FIN-00014 Helsinki, Finland

${ }^{16}$ Joint Institute for Nuclear Research, Dubna, Head Post Office, P.O. Box 79, 101000 Moscow, Russian Federation

${ }^{17}$ Institut für Experimentelle Kernphysik, Universität Karlsruhe, Postfach 6980, D-76128 Karlsruhe, Germany

${ }^{18}$ Institute of Nuclear Physics and University of Mining and Metalurgy, Ul. Kawiory 26a, PL-30055 Krakow, Poland

${ }^{19}$ Université de Paris-Sud, Lab. de l'Accélérateur Linéaire, IN2P3-CNRS, Bât. 200, F-91405 Orsay Cedex, France

${ }^{20}$ School of Physics and Materials, University of Lancaster, Lancaster LA1 4YB, UK

${ }^{21}$ LIP, IST, FCUL - Av. Elias Garcia, 14-1 ${ }^{\circ}$, P-1000 Lisboa Codex, Portugal

${ }^{22}$ Department of Physics, University of Liverpool, P.O. Box 147, Liverpool L69 3BX, UK

${ }^{23}$ LPNHE, IN2P3-CNRS, Universités Paris VI et VII, Tour 33 (RdC), 4 place Jussieu, F-75252 Paris Cedex 05, France

${ }^{24}$ Department of Physics, University of Lund, Sölvegatan 14, S-22363 Lund, Sweden

${ }^{25}$ Université Claude Bernard de Lyon, IPNL, IN2P3-CNRS, F-69622 Villeurbanne Cedex, France

${ }^{26}$ Universidad Complutense, Avda. Complutense s/n, E-28040 Madrid, Spain

${ }^{27}$ Univ. d'Aix - Marseille II - CPP, IN2P3-CNRS, F-13288 Marseille Cedex 09, France

${ }^{28}$ Dipartimento di Fisica, Università di Milano and INFN, Via Celoria 16, I-20133 Milan, Italy

${ }^{29}$ Niels Bohr Institute, Blegdamsvej 17, DK-2100 Copenhagen 0, Denmark

${ }^{30} \mathrm{NC}$, Nuclear Centre of MFF, Charles University, Areal MFF, V Holesovickach 2, 180 00, Praha 8, Czech Republic

${ }^{31}$ NIKHEF-H, Postbus 41882, NL-1009 DB Amsterdam, The Netherlands

${ }^{32}$ National Technical University, Physics Department, Zografou Campus, GR-15773 Athens, Greece

${ }^{33}$ Physics Department, University of Oslo, Blindern, N-1000 Oslo 3, Norway

${ }^{34}$ Dpto. Fisica, Univ. Oviedo, C/P. Pérez Casas, S/N-33006 Oviedo, Spain

${ }^{35}$ Department of Physics, University of Oxford, Keble Road, Oxford OX1 3RH, UK

${ }^{36}$ Dipartimento di Fisica, Università di Padova and INFN, Via Marzolo 8, I-35131 Padua, Italy

${ }^{37}$ Rutherford Appleton Laboratory, Chilton, Didcot OX11 OQX, UK

${ }^{38}$ Dipartimento di Fisica, Università di Roma II and INFN, Tor Vergata, I-00173 Rome, Italy

${ }^{39}$ Centre d'Etudes de Saclay, DSM/DAPNIA, F-91191 Gif-sur-Yvette Cedex, France

${ }^{40}$ Istituto Superiore di Sanità, Ist. Naz. di Fisica Nucl. (INFN), Viale Regina Elena 299, I-00161 Rome, Italy

${ }^{41}$ Instituto de Fisica de Cantabria (CSIC-UC), Avda. los Castros, S/N-39006 Santander, Spain, (CICYT-AEN93-0832)

${ }^{42}$ Inst. for High Energy Physics, Serpukov P.O. Box 35, Protvino, (Moscow Region), Russian Federation

${ }^{43}$ J. Stefan Institute and Department of Physics, University of Ljubljana, Jamova 39, SI-61000 Ljubljana, Slovenia

${ }^{44}$ Fysikum, Stockholm University, Box 6730, S-113 85 Stockholm, Sweden

${ }^{45}$ Dipartimento di Fisica Sperimentale, Università di Torino and INFN, Via P. Giuria 1, I-10125 Turin, Italy

${ }^{46}$ Dipartimento di Fisica, Università di Trieste and INFN, Via A. Valerio 2, I-34127 Trieste, Italy and Istituto di Fisica, Università di Udine, I-33100 Udine, Italy

${ }^{47}$ Univ. Federal do Rio de Janeiro, C.P. 68528 Cidade Univ., Ilha do Fundão BR-21945-970 Rio de Janeiro, Brazil

${ }^{48}$ Department of Radiation Sciences, University of Uppsala, P.O. Box 535, S-751 21 Uppsala, Sweden

${ }^{49}$ IFIC, Valencia-CSIC, and D.F.A.M.N., U. de Valencia, Avda. Dr. Moliner 50, E-46100 Burjassot (Valencia), Spain

${ }^{50}$ Institut für Hochenergiephysik, Österr. Akad. d. Wissensch., Nikolsdorfergasse 18, A-1050 Vienna, Austria

${ }^{51}$ Inst. Nuclear Studies and University of Warsaw, Ul. Hoza 69, PL-00681 Warsaw, Poland

${ }^{52}$ Fachbereich Physik, University of Wuppertal, Postfach 100 127, D-42097 Wuppertal 1, Germany

${ }^{53}$ On leave of absence from IHEP Serpukhov
} 


\section{Introduction}

Direct production of vector meson states $(Q \bar{Q})$ of heavy quarks $Q=c, b$ in hadronic $Z^{0}$ decay is expected to be dominated by two fragmentation processes[1], [2]

- heavy quark fragmentation: $Z^{0} \rightarrow(Q \bar{Q}) Q \bar{Q}$

- gluon fragmentation: $Z^{0} \rightarrow q \bar{q} g^{*}, g^{*} \rightarrow(Q \bar{Q}) g g$

where $q$ represents any quark $u, d, c, s$ or $b$. In perturbative QCD, there is also a shortdistance process (scale $1 / M_{Z^{0}}$ ) of hard gluon radiation off the heavy quarks which can then form a bound state[3]

$$
\text { - } Z^{0} \rightarrow(Q \bar{Q}) g g
$$

This paper describes a search for promptly produced $\psi$ and $\Upsilon$ mesons decaying to an electron or muon pair in hadronic $Z^{0}$ decays at LEP. Such events would signal the presence of direct production processes. The prompt meson states are identified by their leptonic decays: a pair of leptons with an invariant mass compatible with the meson state and produced at the interaction point is a common signature in all direct production processes.

Isolation of leptons with respect to the jet systems and absence of a positive tag for $b$ hadrons in the hadronic jets are good signatures for the gluon processes (2) and (3) because they produce quarkonium states at higher $p_{T}[2]$ and the hadronic system often consists only of light flavour hadrons. Known backgrounds such as $J / \psi$ from bottom decays or events with semileptonic decays of heavy flavour hadrons, possibly with hadrons misidentified as leptons, can be eliminated very efficiently.

The situation is more involved for quark fragmentation processes, which are expected to produce leptons at transverse momentum of the order of $M_{Q \bar{Q}}$ with respect to the primary quark direction, and the accompanying hadronic systems carry the same heavy flavour as the quarks bound within the meson state. The presence of prompt $J / \psi$ production is probed here using the lifetime distribution of the inclusive $J / \psi$ sample, while the search for a prompt $\Upsilon$ signal suggests the use of isolation criteria, because of its higher mass, and of a positive $b$ tag in the accompanying hadronic jets.

QCD based calculations [1] predict probabilities of $2.4 \times 10^{-4}$ for a $J / \psi$ state to be produced in an event of $Z^{0} \rightarrow c \bar{c}$, and of $4.2 \times 10^{-5}$ for an $\Upsilon(1 S)$ state in an event of $Z^{0} \rightarrow b \bar{b}$ (process (1)). For $J / \psi$, the branching fraction through the second process in hadronic $Z^{0}$ decays is $2 \times 10^{-5}[2]$. The branching ratios $\operatorname{Br}\left(Z^{0} \rightarrow(Q \bar{Q}) g g\right)$ are expected to be small, with branching ratios of $4 \times 10^{-7}$ and $2 \times 10^{-6}$ to $J / \psi$ and $\Upsilon(1 S)$, respectively.

Up to now in the LEP data, an upper limit of $7 \times 10^{-4}(90 \% \mathrm{CL})$ has been set for direct production of $J / \psi$ states through the process (2) by the L3 experiment [4]. DELPHI has estimated a primary component of $7.7_{-5.4}^{+6.3}($ stat $) \%$ in the proper time distribution of the inclusive $J / \psi$ sample (Ref. [5]). The inclusive multiplicity of charm quark pairs from gluons has been measured by the OPAL experiment [6] to be $\left.<n_{c \bar{c}}\right\rangle=(2.27 \pm 0.28 \pm 0.41) \times 10^{-2}$ per hadronic $Z^{0}$, in agreement with the predictions from the JETSET 7.3 fragmentation model used. However, preliminary results from the CDF collaboration have reported on an order of magnitude higher rates of direct $J / \psi, \psi(2 S), \Upsilon(1 S)$ and $(2 S)$ production in $p \bar{p}$ collisions [7] compared to initial predictions [1]. Several production mechanisms have been proposed to explain the CDF data $[8]$. 


\section{Detector}

This analysis relies mainly on identification of isolated muons and electrons, reconstructed with precise vertex parameters. Charged and neutral particles are used in defining hadronic jets. The detector components relevant to these aspects of event information are described here. A general description of the DELPHI detector and its performance can be found in $[9]$ and $[10]$.

The microvertex detector (VD) is closest to the interaction point. It has three layers of silicon strip detectors at radii of $6.3,9.0$ and $11.0 \mathrm{~cm}$. Coordinates in the $R \phi$ plane which is transverse to the electron beam direction, $z$, are available at polar angles $\theta$ from $37^{\circ}$ to $143^{\circ}$. The VD points alone provide an impact parameter precision of $24 \mu \mathrm{m}$ for high momentum charged particles. In the data collected in 1994, the $z$ coordinate is measured with a precision comparable to $R \phi$ by the double-sided VD layers. The charged particle trajectories are reconstructed in the DELPHI central tracking system using the Inner Detector, the Time Projection Chamber (TPC) and the Outer Detector which cover polar angles between $30^{\circ}$ and $150^{\circ}$. A precision of $3.5 \%$ has been obtained on the momentum of muons of $45 \mathrm{GeV} / \mathrm{c}$ in the solenoidal magnetic field of $1.2 \mathrm{~T}$. At polar angles $11^{\circ}-33^{\circ}$ and $147^{\circ}-169^{\circ}$ forward drift chambers extend the tracking acceptance.

Electron showers are reconstructed within the polar angles of $42^{\circ}-138^{\circ}$ in the High density Projection Chamber (HPC) with a depth of 17.5 radiation lengths (for $\theta$ near $\left.90^{\circ}\right)$. The electron identification algorithm is based on the shower profile, the ratio of the shower energy to the track momentum $(E / p)$, and the particle mass determination by using the Ring Imaging Cherenkov counters and $d E / d x$ data from the TPC. Within the acceptance of the HPC, 85 to $90 \%$ of the electrons with momenta greater than $2 \mathrm{GeV} / \mathrm{c}$ are identified with a probability of $5 \%$ for hadrons in jets to be misidentified as electrons. The momentum reconstruction of electrons includes corrections for traversing the detector material by using a track refit and detected bremsstrahlung photons. The electromagnetic energy measured in the forward electromagnetic calorimeter (EMF) at polar angles $10^{\circ}-$ $36^{\circ}$ and $144^{\circ}-170^{\circ}$ has been used in the reconstruction of forward jets. The gain in using electrons identified in the EMF would be marginal because of lower acceptance and efficiency, and higher background due to increased detector material in front of it.

Muons are identified as charged particles with associated hits in muon chambers in the outer part of the instrumented hadron calorimeter in the iron magnet yoke. Muon candidates traverse a thickness of 7.5 absorption lengths or more $\left(\theta\right.$ near $\left.90^{\circ}\right)$. Three sets of barrel drift chambers, each with two cell layers with azimuthal overlap, provide threedimensional hit information for muons at polar angles $52^{\circ}<\theta<128^{\circ}$. The hits patterns in two forward muon chamber layers which cover polar angles $9^{\circ}-43^{\circ}$ and $137^{\circ}-171^{\circ}$ are also used. The gap between the barrel and forward muon chambers is covered by streamer tube planes, present since 1994. The muon identification efficiency within the acceptance of the muon chambers is $\sim 90 \%$ at momenta greater than $3 \mathrm{GeV} / \mathrm{c}$ with an estimated probability of $3 \%$ for a hadron in a jet to be misidentified as a muon.

\section{Data Samples}

The analysis is based on data collected with the DELPHI detector at LEP in the period 1991-1994 consisting of about $3.2 \times 10^{6}$ hadronic $Z^{0}$ decays.

A sample of $5.5 \times 10^{6} Z^{0} \rightarrow q \bar{q}$ events generated by the JETSET 7.3 parton shower Monte Carlo [11] with the DELPHI tuning of $b$ and $c$ decays ( $B$ meson lifetime 1.6 ps), followed by the full detector simulation [12] has been used. Specific simulated samples 
of the $b \rightarrow J / \psi X$ channel with varying lifetime, decay and fragmentation characteristics of bottom hadrons have also been used. In addition, simulated samples of four-fermion processes $e^{+} e^{-} \rightarrow l l q \bar{q}[13]$ have been used to describe this expected background. The efficiencies of the selection criteria for the direct production mechanisms have been checked by using fully simulated events. The direct production channel $Z^{0} \rightarrow q \bar{q} g^{*}, g^{*} \rightarrow J / \psi g g$ has been studied using the generator[2]. The heavy quark fragmentation is described in the JETSET 7.3 model with a momentum spectrum which agrees with the prediction[1], while the rate is about a factor of three higher. For the channel $Z^{0} \rightarrow J / \psi(\Upsilon) g g$, a phase space model with JETSET7.3 fragmentation has been developed.

\subsection{General Event Selection}

Charged particles with polar angles between $20^{\circ}$ and $160^{\circ}$, with track length larger than $30 \mathrm{~cm}$ and with momenta greater than $100 \mathrm{MeV} / \mathrm{c}$ are selected. The relative momentum error has to be less than one, and projections of impact parameters with respect to the beam position less than $5 \mathrm{~cm}$ in the transverse plane and $10 \mathrm{~cm}$ in the beam direction are required. Energy clusters in calorimeters, with the cluster energy below $45 \mathrm{GeV}$ and without associated charged particles are taken as neutral particles.

Hadronic $Z^{0}$ events are selected as events with charged multiplicity more than four, with the total charged energy greater than $0.10 \times E_{c m}$, and with the thrust axis satisfying $\left|\cos \theta_{\text {thrust }}\right|<0.95$. A total of 3125150 events have been selected. The selection efficiency is larger than $98 \%$ for hadronic $Z^{0}$ decays. The background from $\tau^{+} \tau^{-}$pairs (and $\gamma \gamma$ collisions to smaller extent), evaluated by simulation of these processes, has been estimated to be $0.7 \%$ in the analyzed sample.

\subsection{Selection of Isolated Lepton Pairs}

Pairs of identified leptons have been considered when

- both lepton momenta are greater than $3 \mathrm{GeV} / \mathrm{c}$ and the sum of the lepton energies is greater than $10 \mathrm{GeV}$;

- the opening angle of the lepton pair is less than $90^{\circ}$;

- the probability for both leptons to come from the same decay vertex in space is greater than $0.1 \%$.

Electrons recognized as photon conversions in the detector material are rejected [10]. For each lepton pair considered, the hadronic system obtained by removing the lepton pair is reconstructed as two jets by using an appropriate value of the $y_{\text {min }}$ parameter [14]. Only pairs with both jet energies above $10 \mathrm{GeV}$ are considered.

The numbers of selected lepton pairs in the invariant mass interval from 2 to $15 \mathrm{GeV} / \mathrm{c}^{2}$ are listed in table 1, labelled as 'selected pairs'. Predictions from simulation of hadronic $Z^{0}$ decays and four-fermion processes are also shown, normalized to the number of hadronic events in real data. The systematic uncertainties in comparing real data with simulation have been studied by relaxing the lepton identification requirements to charged particle-particle and charged particle-lepton pairs in the mass range 2 to $15 \mathrm{GeV} / \mathrm{c}^{2}$ for the real and simulated data. The general agreement leads to estimates of systematic uncertainties summarized in the first and the second items of table 2.

The background to prompt lepton pairs from bottom decay to $J / \psi$, from semileptonic decays of heavy quarks and from hadrons misidentified as leptons, is reduced by requiring isolated lepton pairs and small missing energy for the event by means of the following criteria: 
- the sum of energies of charged and neutral particles in the cones of $20^{\circ}$ half opening angle around the lepton candidates is required to be less than $4 \mathrm{GeV}$;

- leptons are required to have angles $\alpha$ with respect to the jet axes such that $|\cos \alpha(j e t, l)|<0.8$;

- in events with total reconstructed energy less than $0.80 \times E_{c m}$, the angle $\beta$ between the missing momentum vector and the lepton pair momentum is required to be above $60^{\circ}$.

The distributions of these variables are shown in figures 1 and 2 , and the statistics of lepton pairs satisfying these criteria are listed in table 1. A systematic uncertainty of $20 \%$ has been evaluated (table 2). This includes detector and model uncertainties (lepton spectra). The rates of like-signed lepton pairs are low in the real data in agreement with simulation which suggests that the fraction of misidentified isolated leptons is small. (Like-signed lepton pairs due to $B^{0}-\bar{B}^{0}$ mixing are removed by the cut on opening angle of the lepton pair). The lower number of $e^{+} \mu^{-}$pairs relative to the $\mu^{+} \mu^{-}$and $e^{+} e^{-}$ sample in the isolated selection indicates that double semileptonic decays are suppressed in the selected sample. The fact that the missing energy/momentum veto has a small effect on the sample of isolated $l^{+} l^{-}$pairs adds further support that they do not originate from double semileptonic decays.

\subsection{Lepton Pair Decay Lengths and $b$ tagging}

The decay length $d_{x y}$ of the lepton pair is computed as the absolute difference between the fitted di-lepton vertex and the mean beam spot position in the $x y$ plane averaged over periods of the order of one hour of colliding beams. Figure 3 shows the decay length $d_{x y}$ and its precision $\sigma\left(d_{x y}\right)$. A lepton pair is considered to be produced in the primary interaction point (prompt pair) if

$$
d_{x y}<2.5 \sigma\left(d_{x y}\right) \text { and } d_{x y}<d_{x y}^{\max }
$$

where $d_{x y}^{\max }=0.5(1.0) \mathrm{mm}$ is used for muon (electron) pairs with two or more VD points associated to each track and $d_{x y}^{\max }=1.0(2.0) \mathrm{mm}$ for muon (electron) pairs having one or no VD hit association. These criteria take into account the difference in resolutions of muon and electron pairs, and they allow for poorer decay length precision for muons outside the VD acceptance. The upper part of table 3a lists the efficiencies of decay length cuts for selected pairs from simulated $J / \psi$ from decays of $b$-hadrons and from simulated direct processes. The agreement between simulation and real data is shown in the upper part of table 3b. Further checks on two particle and particle-lepton pairs limit the systematic uncertainty to $10 \%$ for the numbers of lepton pairs selected by the decay length criteria (table 2). This estimate includes the uncertainty in the lifetime of $b$ hadrons, as a variation of $8 \%$ in the rate of selected pairs is observed for a change of $0.1 \mathrm{ps}$ in the lifetime of $b$-hadrons in samples of simulated $b \rightarrow J / \psi X$.

The flavour content of the hadronic jets is analyzed with the DELPHI $b$ tagging procedure. The algorithm uses impact parameter significances to compute probability $P_{\mathrm{vtx}}$ of a set of charged particles all to originate from the primary vertex; the probability is computed from tracks with positive lifetime-signed impact parameters. The algorithm has been calibrated for the analyzed data sets with the procedure used in [15]. The veto of bottom events, defined as $P_{\mathrm{vtx}}>0.1$, is used in selecting candidates in all the channels except the bottom fragmentation to $\Upsilon$. In this channel a bottom tag, defined as $P_{\mathrm{Vtx}}<0.1$, is required. $P_{\mathrm{vtx}}$ is always computed from tracks with positive impact 
parameters. The rates of selected particle pairs in real data and simulation agree within $2 \%$, which is assigned as a systematic uncertainty in the $b$ tagging. The lower parts of tables 3a) and b) summarize the expected selection efficiencies when both the decay length criteria and the $b$ veto are imposed.

Thirteen $\mu^{+} \mu^{-}$and six $e^{+} e^{-}$isolated pairs satisfy the decay length criteria. Two $\mu e$ pairs remain out of twelve selected pairs. If, in addition, the $b$ veto is imposed, ten $\mu^{+} \mu^{-}$, five $e^{+} e^{-}$pairs and a $\mu e$ pair remain. In the simulation, $10.3 \pm 3.2$ (stat. and sys.) $l^{+} l^{-}$ $(l=\mu$ or $e)$ pairs with short decay lengths are expected, and $7.3 \pm 2.3$ events remain after the $b$ veto, dominantly from four-fermion events (two thirds) and combinatorial background (one third). The expected number of $J / \psi$ events coming from $b$-hadrons is predicted to be $0.4 \pm 0.1$; the contribution from $\psi(2 S)$ is estimated to be negligible. It should be noted, that the contribution from direct production mechanisms in the general simulated sample (JETSET) is small within the overall systematics and does not need to be subtracted. The background in the di-lepton mass range $M>5 \mathrm{GeV} / \mathrm{c}^{2}$ consists of four-fermion events only, and is $2.5 \pm 0.2$ events.

While the samples of isolated lepton pairs in data and in simulation agreed within statistics before the vertex criteria, and the efficiencies of vertex criteria on larger inclusive samples agree as well, the residual sample of isolated $l^{+} l^{-}$pairs in real data after vertex criteria is slightly higher than expectations. The data and simulation agree very well for the $e \mu$ and $l^{ \pm} l^{ \pm}$pairs. This information is summarized in table 1 . The invariant masses of the selected lepton pairs in data and in simulation (hadronic $Z^{0}$ decays and four-fermion processes added up) are shown in figure 4. A summary of relevant event variables of the candidate events is listed in table 4.

\section{Mass Resolution and the Resonance Hypothesis}

The reconstructed masses of $\mu^{+} \mu^{-}$pairs from real data and simulated $J / \psi$ and $\Upsilon(1 S)$ states are displayed in figure $5 \mathrm{a}$ ) and $\mathrm{c}$ ). The mass windows $2.96-3.24 \mathrm{GeV} / \mathrm{c}^{2}, 3.51-$ $3.85 \mathrm{GeV} / \mathrm{c}^{2}$ contain $90 \%$ of the muon pairs from $J / \psi$ and $\psi(2 S)$, respectively. The interval of $9.20-10.6 \mathrm{GeV} / \mathrm{c}^{2}$ contains $95 \%$ of those from $\Upsilon(1 S), \Upsilon(2 S)$ or $\Upsilon(3 S)$ decays. The mass window of $2.60-3.86 \mathrm{GeV} / \mathrm{c}^{2}$ is estimated to contain $90 \%$ of the electron pairs from $J / \psi, \psi(2 S)$, and the window $7.9-10.8 \mathrm{GeV} / \mathrm{c}^{2} 95 \%$ of $\Upsilon$ states, see figure $5 \mathrm{~b}$ ) and $\mathrm{d}$ ). The wider mass windows are needed for $e^{+} e^{-}$distributions because of bremsstrahlung of electrons.

By comparing the measured invariant masses with the vector meson mass windows, eight events are found to be compatible with being decays of $J / \psi, \psi(2 S)$ or $\Upsilon$ states. There are four $J / \psi$ candidates, two events of $\psi(2 S) \rightarrow l^{+} l^{-}$, and two candidates in the mass window of $\Upsilon$. A typical candidate is displayed in figure 6 . The event rates and the expected background in the mass windows and in the continuum are summarized in table 5. The statistical probability for such a number of events (or more) to appear in mass windows is computed using the prescription[16] (Poisson processes with background). The uncertainty in the estimated background is taken into account by sampling the background with a Gaussian distribution. Probabilities of $5.4 \%, 3.1 \%$ and $1.1 \%$ are found for the data vs. expectations within the $J / \psi, J / \psi+\psi(2 S)$, and $J / \psi+\psi(2 S)+\Upsilon$ windows, respectively. The probability to have more than six events in the continuum is $25 \%$. The small observed excess thus has a tendency to be related to the meson mass windows rather than to the continuum. There is no significant excess in any individual window, however. 


\section{Sensitivity to Standard Production Mechanisms, and Upper Limits}

The efficiencies of the selection criteria for the production mechanisms $(1-3)$ have been checked by using fully simulated events (see table 6 ). For $J / \psi$ from charm and gluon fragmentation, and for the hard gluon radiation, the decay length cuts and the $b$ veto are imposed in estimating the efficiencies. The decay length cuts and $b$ tag are required for the channel $Z^{0} \rightarrow b \bar{b} \Upsilon$. The uncertainties shown are due to simulation statistics, only.

As expected, the selection has a very low efficiency for charm fragmentation into $\mathrm{J} / \psi$ due to cuts on isolation and on jet-lepton angles. This channel is better constrained by using the inclusive $J / \psi$ proper time distribution of the earlier DELPHI analysis of [5]. The prompt component of $7.7_{-5.4}^{+6.3}($ stat $) \%$ is combined with the world average values [16] for relevant branching ratios of $Z^{0} \rightarrow b \bar{b}$ and $b \rightarrow J / \psi$. As the prompt component is compatible with zero, the result can be expressed as $90 \%$ confidence level upper limit as listed in table 5 .

The observed candidates, the background estimates, the efficiency estimates and the leptonic branching ratios are used for the processes (2) and (3) as follows. As the number of observed candidates is not significantly above expectations in any mass window, the result is best expressed as $90 \%$ confidence level upper limits. The upper limits for signal events in the mass windows of $J / \psi$ and $\Upsilon(1 S),(2 S),(3 S)$ are computed analogously to the probability estimates of the previous section. In computing the limit for the final state $b \bar{b} \Upsilon$, no candidates are assumed as the observed hadronic systems in the selected events are unlikely $b$ jets (the probability of two $b \bar{b} \Upsilon$ candidates to satisfy $b$ veto is less than $2 \%$ ). The uncertainties in efficiency estimates are taken into account by shifting them down by one standard deviation. The upper limits are listed in table 5, which also includes branching ratio estimates if the events are taken as signal. The branching ratios are computed from the observed number of candidates after subtracting the expected background.

\section{Summary}

A data sample of more than 3 million hadronic $Z^{0}$ events from the DELPHI detector has been analyzed to search for direct production of $J / \psi, \psi(2 S)$ and $\Upsilon(1 S),(2 S)$ or $(3 S)$ states. Kinematical cuts and vertex criteria have been used to select events with a lepton pair where the background from weak decays and the continuum spectrum has been highly suppressed.

Upper limits are set for the expected strong production mechanisms of $J / \psi, \psi(2 S)$ and $\Upsilon$ states at $90 \%$ confidence level

- $\operatorname{Br}\left(Z^{0} \rightarrow c \bar{c} J / \psi\right) / B r\left(Z^{0} \rightarrow\right.$ hadrons $)<6 \times 10^{-4}$

- $\operatorname{Br}\left(Z^{0} \rightarrow q \bar{q} g^{*}, g * \rightarrow J / \psi g g\right) / B r\left(Z^{0} \rightarrow\right.$ hadrons $)<6 \times 10^{-4}$

- $\operatorname{Br}\left(Z^{0} \rightarrow J / \psi g g\right) / B r\left(Z^{0} \rightarrow\right.$ hadrons $)<0.9 \times 10^{-4}$

- $\operatorname{Br}\left(Z^{0} \rightarrow q \bar{q} g^{*}, g * \rightarrow \psi(2 S) g g\right) / B r\left(Z^{0} \rightarrow\right.$ hadrons $)<29 \times 10^{-4}$

- $\operatorname{Br}\left(Z^{0} \rightarrow \psi(2 S) g g\right) / \operatorname{Br}\left(Z^{0} \rightarrow\right.$ hadrons $)<3.9 \times 10^{-4}$

- $\operatorname{Br}\left(Z^{0} \rightarrow b \bar{b} \Upsilon\right) / B r\left(Z^{0} \rightarrow\right.$ hadrons $)<9 \times 10^{-4}$

- $\operatorname{Br}\left(Z^{0} \rightarrow \Upsilon g g\right) / \operatorname{Br}\left(Z^{0} \rightarrow\right.$ hadrons $)<2 \times 10^{-4}$

The limits are either new or improve earlier results. The results are extracted in the presence of a small excess over the expectations when the candidate events are summed 
up in the mass windows of $J / \psi, \psi(2 S), \Upsilon$. This excess has a probability of about $1 \%$ to be a statistical fluctuation. Apart from a statistical fluctuation, the events may arise from an unaccounted or underestimated source. For instance, the resonance contributions in the 4-fermion processes are not considered in simulation. Their rate, however, is expected to be small [17], about $10 \%$ correction to the estimate from the 4 -fermion continuum part within the $J / \psi$ mass window, and as such not sufficient to explain the fluctuation. Among the direct strong production mechanisms studied here, the gluon processes come the closest in interpreting the data. For example, taking the data as a signal of $J / \psi$

from gluon fragmentation would suggest rates which are a factor of ten above the initial predictions [2].

\section{Acknowledgements}

We are greatly indebted to our technical collaborators and to the funding agencies for their support in building and operating the DELPHI detector, and to the members of the CERN-SL Division for the excellent performance of the LEP collider. 


\section{References}

[1] E. Braaten et al. Phys. Rev. D48 (1993) 4230. (agrees with an earlier computation of V. Barger et al. Phys. Rev. D41 (1990) 1541).

[2] K. Hagiwara et al. Phys. Lett. B267 (1991) 527, with erratum in Phys. Lett. B316 (1993) 631; E. Braaten and T.C. Yuan, Phys. Rev. Lett. 71 (1993) 1673, and Phys. Rev. D50 (1994) 3176.

[3] W.Y. Keung, Phys. Rev. D23 (1981) 2072.

[4] O. Adriani et al. (L3 Collaboration) Phys. Lett. B288 (1992) 412.

[5] P. Abreu et al. (DELPHI Collaboration) Phys. Lett. B341 (1994) 109.

[6] R. Akers et al. (OPAL Collaboration) Phys. Lett. B353 (1995) 595.

[7] T. Daniels representing the CDF Collaboration, " $J / \psi, \psi^{\prime} \rightarrow \mu^{+} \mu^{-}$and $B \rightarrow J / \psi, \psi^{\prime}$ Cross Sections", DPF'94 Conference, Albuquerque, U.S.A., August 1994; V. Papadimitriou " $\Upsilon$ Production at CDF", ibid.

[8] E. Braaten, M. A. Donchevski, S. Fleming, M. L. Mangano, Phys. Lett. B333 (1994), 548; D. P. Roy, K. Sridhar, Phys. Lett. B341 (1995), 413; P. Cho, M. B. Wise, Phys. Lett. B346 (1995), 129; E. Braaten, S. Fleming, Phys. Rev. Lett. 74 (1995) 3327; K. Cheung, "Recent Progress on Perturbative QCD Fragmentation Functions", PASCOS/HOPKINS 1995 Symposium,Baltimore,U.S.A., March 1995, CPP-95-8; M. Cacciari et al. "Charmonium Production at the Tevatron" CERN-TH/95-129, May 1995.

[9] P. Aarnio et al. (DELPHI Collaboration), Nucl. Instr. and Meth. A303(1991) 233.

[10] P. Abreu et al. (DELPHI Collaboration), "Performance of the DELPHI Detector", contribution to the EPS-HEP 95 conference, Brussels, August 1995.

[11] T. Sjöstrand, Computer Physics Comm. 39 (1986) 347; T. Sjöstrand and M. Bengtsson, Computer Physics Comm. 43 (1987) 367.

[12] DELSIM Reference Manual, DELPHI 89-98 PROG 100, Geneva, July 1989.

[13] R. Contri and S. Simonetti, "An External Generator for Four Fermion Processes", DELPHI Note, 91-24, PROG 162, May 1991.

[14] W. Bartl et al. (JADE Collaboration), Z. Phys. C33 (1986) 23.

[15] P. Abreu et al. (DELPHI Collaboration), Zeit. Phys. C65 (1995) 555; G. Borisov, "Lifetime Tag in Events with B hadrons with the DELPHI Detector", IHEP (Protvino) 94-98 (1994).

[16] The Particle Data Group, Phys. Rev. D50 (1994) 1173.

[17] A.H. Hoang et al. ,Phys. Lett. B325 (1994), 495, erratum ibid. B327 (1994), 439; S. Fleming, Phys. Rev. D50 (1994) 5808. 


\begin{tabular}{|c|c|c|c|c|c|}
\hline selection & Real +- & $\operatorname{Sim}\left(Z^{0}\right)+-$ & $\operatorname{Sim}(4-f)+-$ & Real \pm \pm & Sim. \pm \pm \\
\hline \multicolumn{6}{|c|}{ a) \# selected pairs: } \\
\hline$\mu \mu$ & 764 & $860 \pm 22$ & $11 \pm 2.5$ & 224 & $219 \pm 11$ \\
\hline$e e$ & 450 & $450 \pm 46$ & $5 \pm 1.5$ & 171 & $168 \pm 16$ \\
\hline$e \mu$ & 759 & $801 \pm 20$ & - & 369 & $353 \pm 13$ \\
\hline \multicolumn{6}{|c|}{ b) \# isolated pairs } \\
\hline$\mu \mu$ & 20 & $12 \pm 3$ & $5.1 \pm 1.7$ & 2 & $1.7 \pm 1.0$ \\
\hline$e e$ & 11 & $5.2 \pm 1.7$ & $2.9 \pm 1.3$ & 0 & 0.0 \\
\hline$e \mu$ & 12 & $8.0 \pm 2.1$ & - & 3 & $2.2 \pm 1.1$ \\
\hline \multicolumn{6}{|c|}{ c) \# pairs with short decay lengths } \\
\hline$\mu \mu$ & 13 & $2.5 \pm 0.7$ & $4.2 \pm 0.7$ & 0 & $0.63 \pm 0.37$ \\
\hline$e e$ & 6 & $1.3 \pm 0.5$ & $2.3 \pm 0.5$ & 0 & 0.0 \\
\hline$e \mu$ & 2 & $4.2 \pm 1.1$ & - & 1 & $1.1 \pm 0.57$ \\
\hline \multicolumn{6}{|c|}{ d) \# pairs with short decay lengths and light flavour jets } \\
\hline$\mu \mu$ & 10 & $1.3 \pm 0.5$ & $3.5 \pm 0.5$ & 0 & $0.34 \pm 0.20$ \\
\hline$e e$ & 5 & $0.8 \pm 0.3$ & $1.7 \pm 0.3$ & 0 & 0.0 \\
\hline$e \mu$ & 1 & $2.2 \pm 0.6$ & - & 0 & $0.33 \pm 0.17$ \\
\hline
\end{tabular}

Table 1: Summary of events rates satisfying the selection criteria, showing, from left to right, opposite-sign rates for data, simulated $Z^{0}$ hadronic events and simulated 4-fermion processes, and like-sign rates for data and simulation. The simulation results are normalized to the number of hadronic events in real data. The errors in simulated data are statistical, the systematic uncertainty is $30 \%$.

\begin{tabular}{|l|l|c|}
\hline Selection & Source of uncertainty & Uncertainty \\
\hline Selected track pairs & $\begin{array}{l}\text { momentum spectra, } \\
\text { track reconstruction and vertex fit } \\
\text { efficiencies and misidentification } \\
\text { Isolation criteria } \\
\text { jet reconstruction, } \\
\text { semileptonic decay properties, } \\
J / \psi \text { decay properties } \\
b \text {-hadron lifetimes, } \\
\text { vertex reconstruction }\end{array}$ & $12 \%$ \\
Simulation statistics & & $20 \%$ \\
\hline Total (quadratic sum) & & $10 \%$ \\
\hline
\end{tabular}

Table 2: Summary of uncertainties in the background estimate for events in the mass windows $J / \psi, \psi(2 S)$ and $\Upsilon(1 S),(2 S),(3 S)$. The systematic uncertainties are estimated as differences in the rates of lepton-lepton, particle-lepton and particle-particle pairs in the real and simulation data. The total uncertainty is the quadratic sum of the individual contributions. 


\begin{tabular}{|r|rc|c|}
\hline \multicolumn{4}{|c|}{ a) Selected pairs from simulation: } \\
\hline criterion & channel: & direct $c \rightarrow J / \psi$ & $b \rightarrow J / \psi$ \\
\hline decay length & $\mu \mu$ & $0.83 \pm 0.04$ & $0.18 \pm 0.04$ \\
& $e e$ & $0.77 \pm 0.10$ & $0.19 \pm 0.04$ \\
\hline and $b$ veto & $\mu \mu$ & $0.69 \pm 0.05$ & $0.082 \pm 0.007$ \\
& $e e$ & $0.59 \pm 0.12$ & $0.095 \pm 0.009$ \\
\hline
\end{tabular}

\begin{tabular}{|r|rcc|cc|}
\hline \hline b) Selected pairs: & \multicolumn{4}{|l|}{} \\
\hline criterion & sample: & real +- & sim +- & real \pm \pm & $\operatorname{sim} \pm \pm$ \\
\hline decay length & $\mu \mu$ & $0.21 \pm 0.02$ & $0.21 \pm 0.01$ & $0.42 \pm 0.05$ & $0.37 \pm 0.03$ \\
& $e e$ & $0.21 \pm 0.03$ & $0.25 \pm 0.02$ & $0.32 \pm 0.05$ & $0.41 \pm 0.03$ \\
& $e \mu$ & $0.28 \pm 0.02$ & $0.27 \pm 0.01$ & $0.34 \pm 0.04$ & $0.41 \pm 0.02$ \\
\hline and $b$ veto & $\mu \mu$ & $0.11 \pm 0.01$ & $0.11 \pm 0.01$ & $0.19 \pm 0.03$ & $0.20 \pm 0.02$ \\
& $e e$ & $0.15 \pm 0.02$ & $0.13 \pm 0.01$ & $0.21 \pm 0.03$ & $0.28 \pm 0.03$ \\
& $e \mu$ & $0.11 \pm 0.01$ & $0.12 \pm 0.01$ & $0.15 \pm 0.02$ & $0.23 \pm 0.02$ \\
\hline
\end{tabular}

Table 3: Efficiencies of the decay vertex and $b$ tagging criteria.

\begin{tabular}{|c|c|c|c|c|c|c|c|}
\hline leptons & $\begin{array}{l}E_{1+2} \\
\mathrm{GeV}\end{array}$ & $\begin{array}{c}p_{1+2}^{T} \\
\mathrm{GeV} / \mathrm{c}\end{array}$ & $\begin{array}{c}M_{l, l} \\
\mathrm{GeV} / \mathrm{c}^{2}\end{array}$ & $\begin{array}{l}d_{x y} \\
\mu \mathrm{m}\end{array}$ & $P_{\mathrm{vtx}}$ & $\begin{array}{c}E_{\text {rec }} / \\
E_{\mathrm{cm}}\end{array}$ & $\begin{array}{c}\alpha_{p_{\text {miss }}, p_{11}} \\
\operatorname{dgr}\end{array}$ \\
\hline$\mu^{+} \mu^{-}$ & 10 & 8 & 2.88 & $120 \pm 160$ & 1.00 & 0.94 & 61 \\
\hline$\mu^{+} \mu^{-}(J / \psi)$ & 15 & 12 & 2.99 & $350 \pm 156$ & 0.65 & 0.75 & 120 \\
\hline$\mu^{+} \mu^{-}(J / \psi)$ & 14 & 9 & 3.04 & $220 \pm 480$ & 0.70 & 1.05 & 122 \\
\hline$\mu^{+} \mu^{-}$ & 24 & 15 & 3.40 & $270 \pm 180$ & 0.11 & 0.63 & 144 \\
\hline$\mu^{+} \mu^{-}(\psi(2 S))$ & 12 & 11 & 3.53 & $350 \pm 320$ & 0.11 & 0.88 & 87 \\
\hline$\mu^{+} \mu^{-}$ & 10 & 7 & 6.04 & $70 \pm 180$ & 0.70 & 0.85 & 53 \\
\hline$\mu^{+} \mu^{-}$ & 16 & 9 & 6.63 & $30 \pm 45$ & 0.67 & 0.60 & 92 \\
\hline$\mu^{+} \mu^{-}$ & 35 & 15 & 8.64 & $140 \pm 140$ & 0.97 & 1.04 & 73 \\
\hline$\mu^{+} \mu^{-}(\Upsilon(2 S))$ & 41 & 15 & 9.77 & $260 \pm 190$ & 0.80 & 0.97 & 144 \\
\hline$\mu^{+} \mu^{-}(\Upsilon(2 S))$ & 23 & 18 & 9.96 & $860 \pm 660$ & 1.00 & 0.88 & 130 \\
\hline$e^{+} e^{-}$ & 12 & 6 & 2.42 & $40 \pm 120$ & 0.49 & 0.58 & 154 \\
\hline$e^{+} e^{-}$ & 11 & 10 & 2.44 & $80 \pm 185$ & 0.16 & 0.81 & 99 \\
\hline$e^{+} e^{-}(J / \psi)$ & 21 & 11 & 2.74 & $490 \pm 260$ & 0.78 & 0.89 & 55 \\
\hline$e^{+} e^{-}(J / \psi)$ & 18 & 15 & 3.05 & $70 \pm 150$ & 0.54 & 0.60 & 148 \\
\hline$e^{+} e^{-}(\psi(2 S))$ & 21 & 13 & 3.47 & $180 \pm 170$ & 0.28 & 0.88 & 46 \\
\hline$\mu e$ & 12 & 7 & 4.15 & $90 \pm 150$ & 0.68 & 0.83 & 148 \\
\hline
\end{tabular}

Table 4: Selected isolated prompt lepton pairs with light flavour jets. The pairs compatible with quarkonium states are indicated. $E_{1+2}$ and $p_{1+2}^{T}$ are the energy sum of the reconstructed leptons and the transverse component of the momentum vector with respect to the closest jet. $M_{l, l}$ is the invariant mass of the fitted lepton system with $1 \sigma$ uncertainty for muon pairs extracted from the fit covariance matrix. For electrons an estimate of the $68 \%$ probability interval is used. $P_{\mathrm{vtx}}$ is explained in the text. The last two columns list the total reconstructed energy and the angle between the missing momentum and the lepton pair momentum vectors. 


\begin{tabular}{|c|c|c|c|c|c|c|c|c|}
\hline \multirow[t]{2}{*}{ channel } & \multirow[t]{2}{*}{$\begin{array}{l}\text { Pred. } \\
\text { /evts }\end{array}$} & \multicolumn{4}{|c|}{$\begin{array}{l}\text { Real prompt pairs } \\
b \text { veto } b \text { tag }\end{array}$} & $\begin{array}{c}\text { Background } \\
/ \text { evts }\end{array}$ & \multirow{2}{*}{$\begin{array}{c}B R_{\text {rate }} \\
\times 10^{-4} \\
/ Z^{0}(\text { had })\end{array}$} & \multirow{2}{*}{$\begin{array}{l}B R_{\text {limit }} \\
\times 10^{-4} \\
/ Z^{0}(\text { had })\end{array}$} \\
\hline & & $\mu \mu$ & $e e$ & $\mu \mu$ & $e e$ & $\mu \mu+e e$ & & \\
\hline$c \bar{c} \rightarrow J / \psi X$ & 0.03 & \multicolumn{5}{|c|}{ incl. lifetime distribution used[5] } & $3.1_{-2.1}^{+2.5}$ & 6.2 \\
\hline$g^{*} \rightarrow J / \psi g g$ & $0.25\}$ & $?$ & 2 & 0 & $n$ & $12+04($ b veto $)$ & $\int 2.2 \pm 1.6$ & 5.9 \\
\hline$Z^{0} \rightarrow g g J / \psi$ & $0.03\}^{3}$ & & & 0 & & $1.2>3$ & $\{0.32 \pm 0.24$ & 0.87 \\
\hline $\begin{array}{l}g^{*} \rightarrow \psi(2 S) g g \\
Z^{0} \rightarrow g g \psi(2 S)\end{array}$ & $\left.\begin{array}{l}- \\
-\end{array}\right\}$ & 1 & 1 & 0 & 0 & $0.96 \pm 0.31$ ( $b$ veto $)$ & $\left\{\begin{array}{l}6.0 \pm 4.7 \\
0.89 \pm 0.70\end{array}\right.$ & $\begin{array}{l}29 \\
3.9\end{array}$ \\
\hline $\begin{array}{l}b \bar{b} \rightarrow \Upsilon X \\
Z^{0} \rightarrow a q \Upsilon\end{array}$ & $\left.\begin{array}{l}0.03 \\
0.06\end{array}\right\}$ & 2 & 0 & 0 & 0 & $\left\{\begin{array}{l}0.12 \pm 0.04(b \text { tag }) \\
0.47 \pm 0.14(b \text { veto })\end{array}\right.$ & $\begin{array}{l}0 \\
0.48 \pm 0.46\end{array}$ & $\begin{array}{l}8.7 \\
1.7\end{array}$ \\
\hline continuum & - & 5 & 2 & 3 & 1 & $4.8 \pm 1.5$ ( $b$ veto $)$ & - & - \\
\hline
\end{tabular}

Table 5: Summary of predictions, data and upper limits. Column 'Pred.' gives predictions from models [1] [2] and [3]. 'Real prompt pairs' gives the numbers of candidate events in mass windows of $J / \psi, \psi(2 S)$ and $\Upsilon$. Numbers of prompt $\mu^{+} \mu^{-}$and $e^{+} e^{-}$pairs are shown, with the additional conditions on $b$ veto or tag. Columns ' $B R_{\text {rate }}$ ' and ' $B R_{\text {limit }}$ ' give branching ratio estimates (see text) and $90 \%$ c.l. upper limits.

\begin{tabular}{|r|c|c|}
\hline Process & \multicolumn{2}{|c|}{ Efficiencies (\%) } \\
& $\mu \mu$ & $e e$ \\
\hline$c \bar{c} \rightarrow J / \psi X$ & $0.2 \pm 0.2$ & $<0.75(90 \% \mathrm{CL})$ \\
$g^{*} \rightarrow J / \psi g g$ & $4.1 \pm 0.5$ & $2.5 \pm 0.3$ \\
$Z^{0} \rightarrow g g J / \psi$ & $28 \pm 2$ & $17 \pm 2$ \\
$b \bar{b} \rightarrow \Upsilon X$ & $2.8 \pm 1.0$ & $1.7 \pm 0.6$ \\
$Z^{0} \rightarrow g g \Upsilon$ & $29 \pm 3$ & $11 \pm 3$ \\
\hline
\end{tabular}

Table 6: Efficiencies of the selection criteria for direct production channels. 

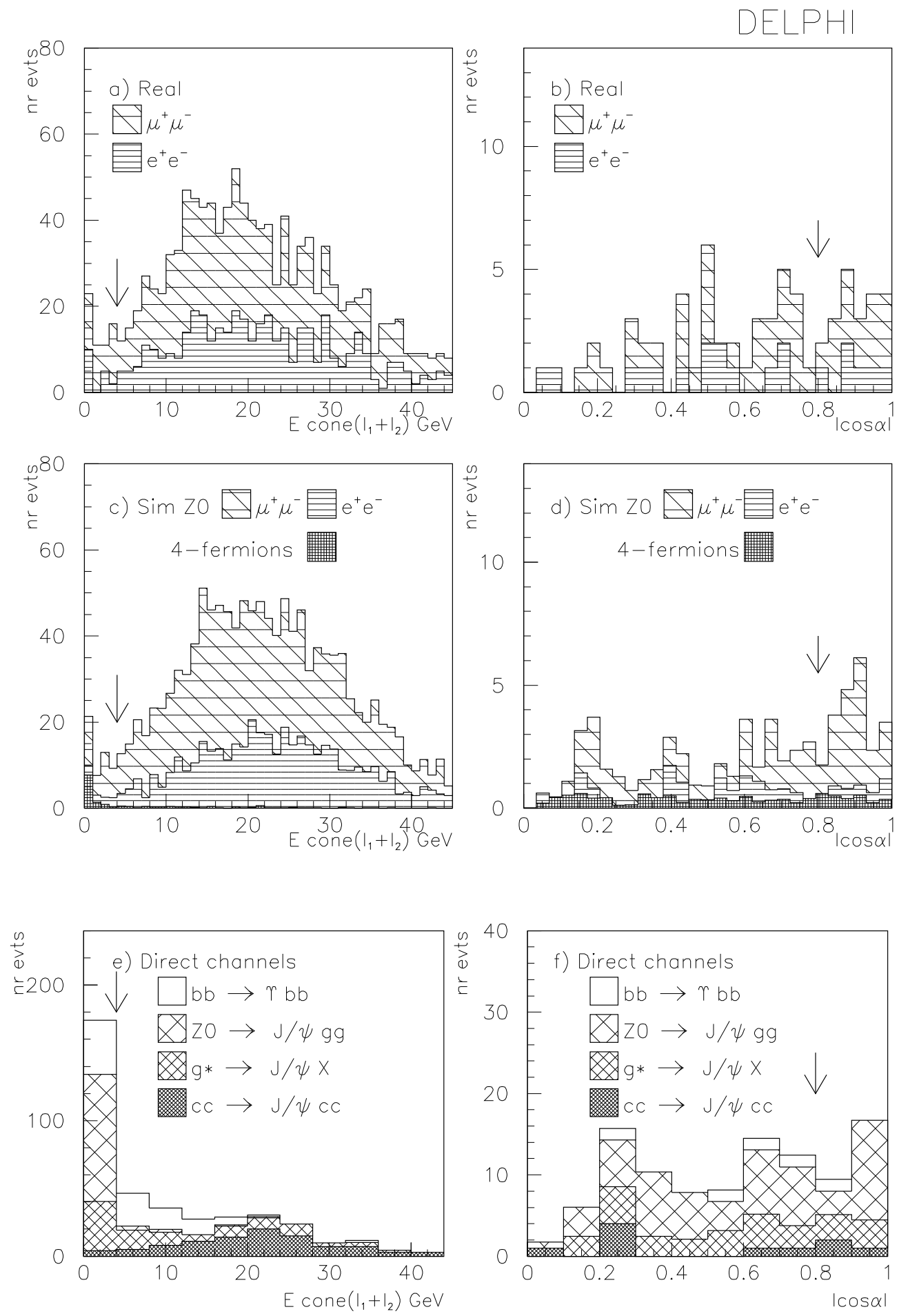

Figure 1: Distributions of the isolation variables: a) cone energy sum for real $\mu^{+} \mu^{-}$and $e^{+} e^{-}$pairs, b) the smallest angle of a lepton with respect to the jet directions for real pairs satisfying the cone energy cut, c) cone energy sum for simulated $Z^{0}$ and 4 -fermion events, d) the smallest angle of a lepton with respect to the jet directions for simulated $Z^{0}$ and 4 -fermion events satisfying the cone energy cut. The distributions from direct production models are shown in e) and $\mathrm{f}$ ) (with arbitrary normalization). Arrows indicate the maximum values allowed for events to be selected. 

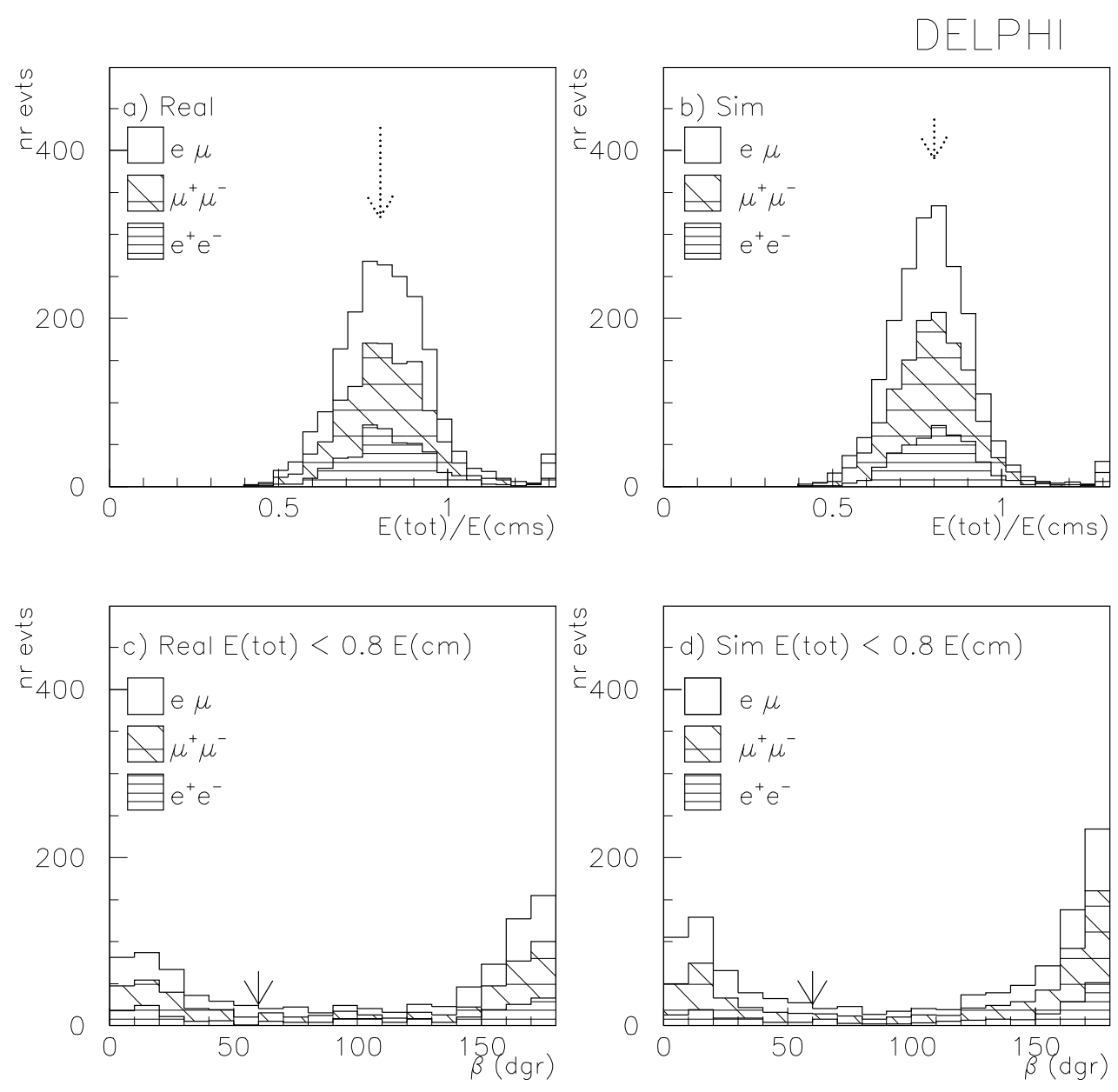

Figure 2: Distributions of the missing energy/momentum variables. a) Total energy normalized to $E_{\mathrm{cm}}$ in events with energetic lepton pairs. c) Angle between the missing momentum and the lepton pair momentum vectors, for events with total energies below $0.8 \times E_{\mathrm{cm}}\left(\right.$ dotted arrow in a)). b) and d) are as a) and c) but for simulated $Z^{0}$ events normalized to the real events. 

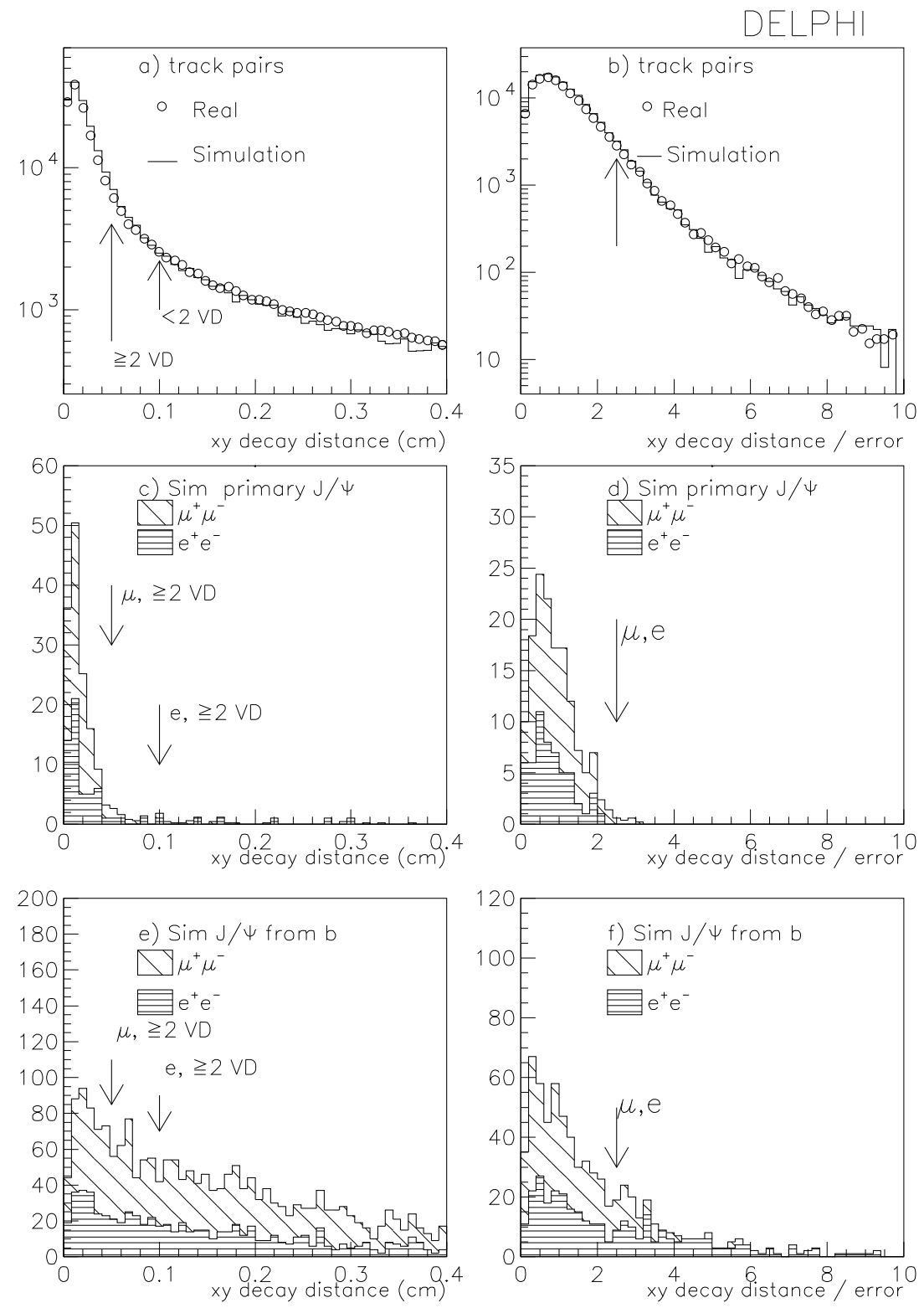

Figure 3: a) Decay length distributions for charged particle pairs satisfying the event selection criteria (nonisolated) in real data (circles) and simulation (histogram). b) as a) but for decay lengths normalized by their errors. The arrows show the maximum values allowed for selected pairs, with two or more associated VD points (' $\geq 2 \mathrm{VD}$ ') or less than two associated VD points (' $<2 \mathrm{VD}$ '). c) Decay length distributions for simulated primary $J / \psi \rightarrow \mu^{+} \mu^{-}$and $e^{+} e^{-}$events. d) Distributions of decay lengths normalized by their errors for the subsample of $\mathrm{c}$ ) within the cut on decay length. e)-f) as c)-d) but for $J / \psi$ from $b$ decays - relative normalization to primary $J / \psi$ is arbitrary. The arrows show the maximum values allowed for selected muon (' $\mu$ ') and electron (' $e$ ') pairs. 

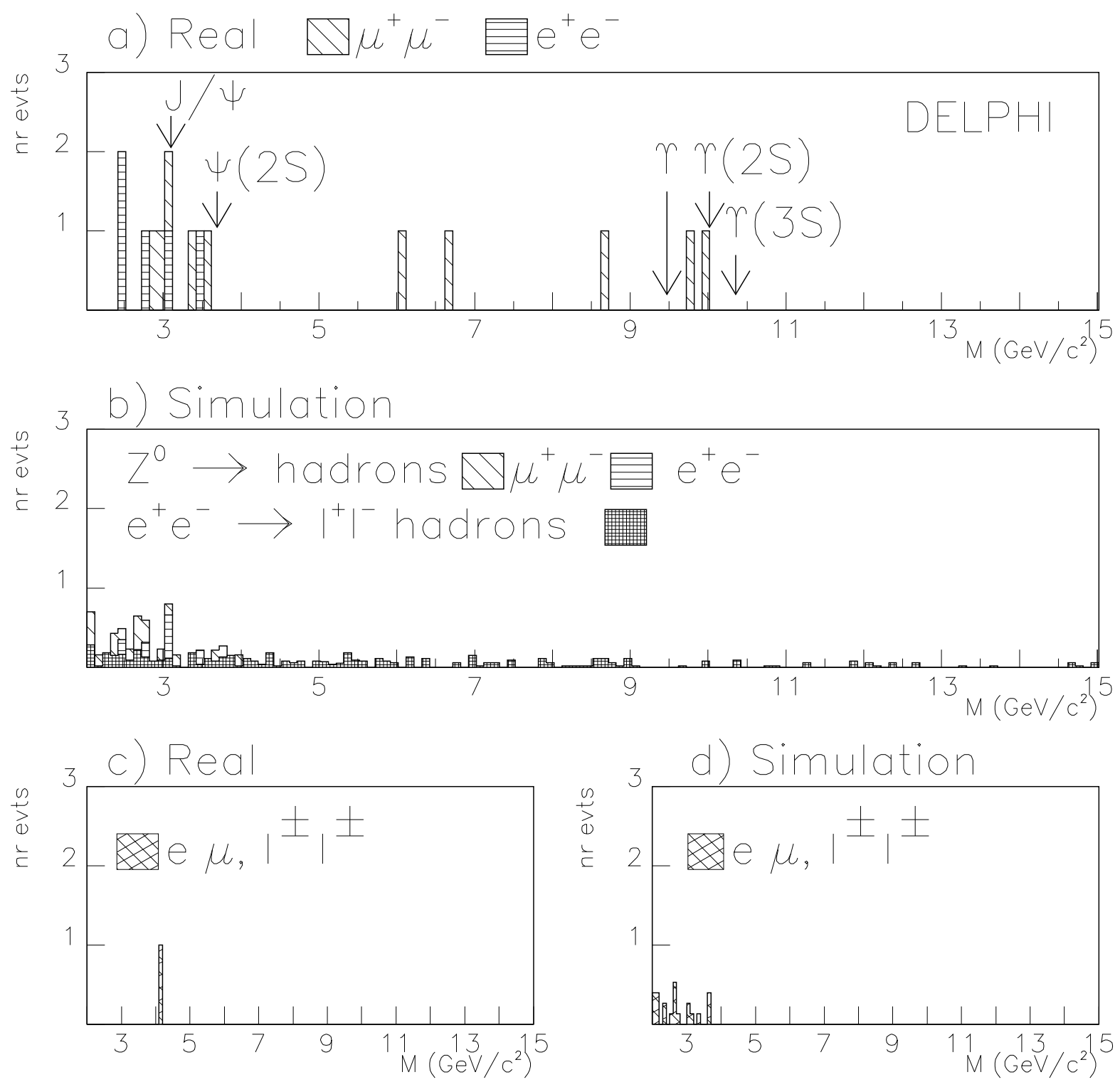

Figure 4: Invariant mass distributions of isolated $\mu^{+} \mu^{-}$and $e^{+} e^{-}$pairs for (a) real data, and b) simulated hadronic $Z^{0}$ decays and process $e^{+} e^{-} \rightarrow l^{+} l^{-} q \bar{q}$. The real $e \mu$ and like-sign lepton pairs are shown in c), and the simulated ones in d). All events satisfy the event selection with cuts on decay lengths and satisfy the $b$ veto. 

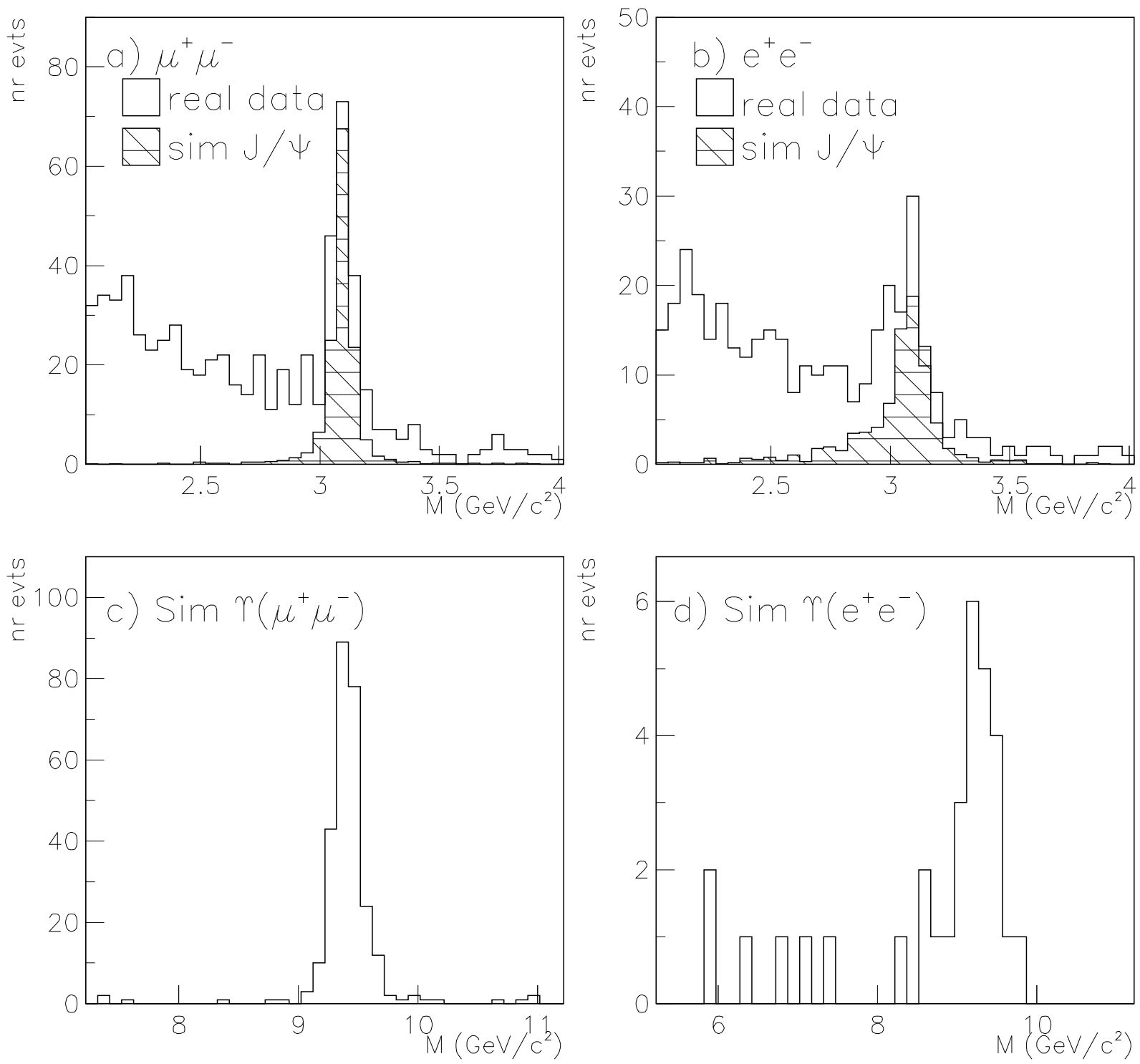

Figure 5: Reconstructed $J / \psi$ and $\Upsilon(1 S)$ masses using their leptonic decays. a) Selected $\mu^{+} \mu^{-}$pairs in real data (see table 1 ) and in simulation (hatched). The mass window $2.96-3.24 \mathrm{GeV} / \mathrm{c}^{2}$ contains $90 \%$ of the simulated $J / \psi$. b) Same as a) for $e^{+} e^{-}$pairs. The $90 \%$ mass window is $2.60-3.26 \mathrm{GeV} / \mathrm{c}^{2}$. c) Simulated $\Upsilon(1 S) \rightarrow \mu^{+} \mu^{-}$. The mass window $9.2-10.6 \mathrm{GeV} / \mathrm{c}^{2}$ contains $95 \%$ of the simulated $\Upsilon(1 S, 2 S, 3 S) \rightarrow \mu^{+} \mu^{-}$. d) Same as c) for $e^{+} e^{-}$. The $95 \%$ mass window is $7.9-10.8 \mathrm{GeV} / \mathrm{c}^{2}$. 


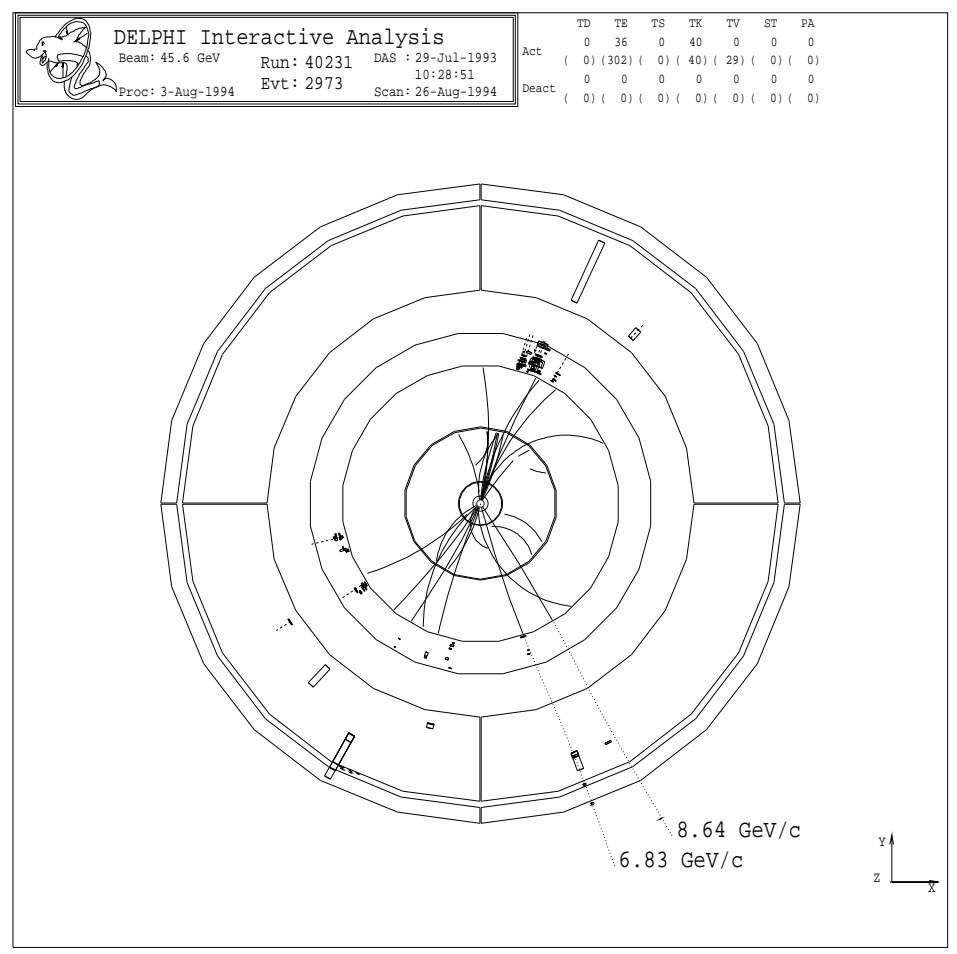

Figure 6: An isolated $\mu^{+} \mu^{-}$pair in a hadronic event, $M_{\mu^{+} \mu^{-}}=2.99 \pm 0.03 \mathrm{GeV} / \mathrm{c}^{2}$, compatible with a $J / \psi$ decay. 